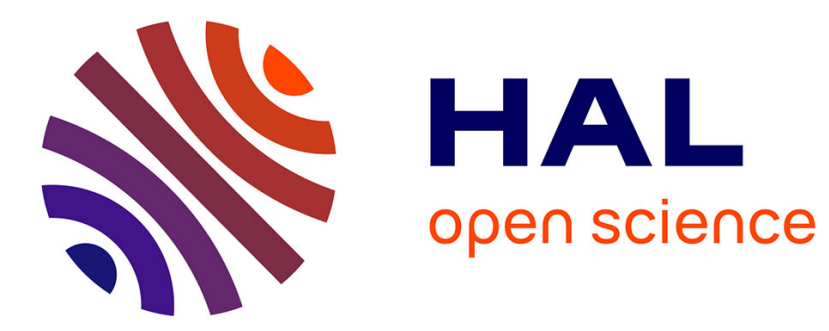

\title{
Rhodium-Catalyzed 1,4-Addition of Arylboronic Acids to 3-Benzylidene-1H-pyrrolo[2,3-b]pyridin-2(3H)-one Derivatives
}

Cécile Croix, Gildas Prié, Charlotte Chaulet, Marie-Claude Viaud-Massuard

\section{- To cite this version:}

Cécile Croix, Gildas Prié, Charlotte Chaulet, Marie-Claude Viaud-Massuard. Rhodium-Catalyzed 1,4-Addition of Arylboronic Acids to 3-Benzylidene-1H-pyrrolo[2,3-b]pyridin-2(3H)-one Derivatives. Journal of Organic Chemistry, 2015, 80 (6), pp.3264-3269. 10.1021/jo502784h . hal-02903786

\section{HAL Id: hal-02903786 \\ https://hal.science/hal-02903786}

Submitted on 21 Jul 2020

HAL is a multi-disciplinary open access archive for the deposit and dissemination of scientific research documents, whether they are published or not. The documents may come from teaching and research institutions in France or abroad, or from public or private research centers.
L'archive ouverte pluridisciplinaire HAL, est destinée au dépôt et à la diffusion de documents scientifiques de niveau recherche, publiés ou non, émanant des établissements d'enseignement et de recherche français ou étrangers, des laboratoires publics ou privés. 


\title{
Rhodium-Catalyzed 1,4-Addition of Arylboronic Acids to 3-Benzylidene-1H-pyrrolo[2,3-b]pyridin-2(3H)-one Derivatives
}

\author{
Cécile Croix, Gildas Prié, Charlotte Chaulet, and Marie-Claude Viaud-Massuard* \\ GICC UMR 7292, Equipe 4 “Innovation Moléculaire et Thérapeutique”, Université de Tours, Labex SYNORG, Faculté de \\ Pharmacie, 31 avenue Monge, 37200 Tours, France
}

Supporting Information

ABSTRACT: 7-Azaindoles are versatile building blocks, especially in medicinal chemistry, where they serve as bioisosteres of indoles or purines. Herein, we present a novel rhodium-catalyzed asymmetric 1,4-addition of arylboronic acids to 3-benzylidene- $1 H$-pyrrolo[2,3- $b]$ pyridin-2(3H)-ones, as these substrates are exocyclic methylene lactamyl Michael acceptors. Ten new original derivatives of $1 H$-pyrrolo[2,3b] pyridin-2(3H)-one have been obtained.
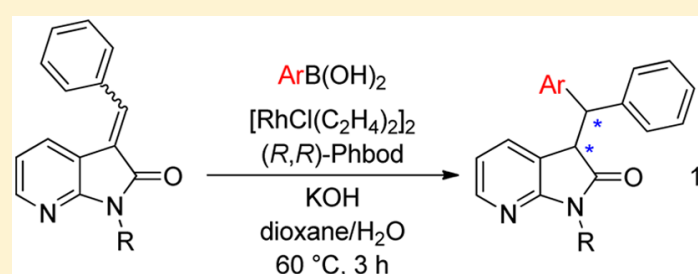

15 examples
$\mathrm{B}$ ecause of their specific chemical behavior, 7-azaindole (1H-pyrrolo[2,3-b] pyridine) derivatives have become an important subject of several synthetic studies. Moreover, due to their particular biological activity, interest in the synthesis of compounds derived from 7-azaindole has arisen in numerous recent pharmacological programs within diverse therapeutic areas, ${ }^{1}$ where they serve as bioisosteres of indole, oxoindole, or purine ring systems. Consequently, as a part of our medicinal chemistry research program, we have been interested in the preparation of new 7-azaoxindole compounds.

The 1,4-conjugate addition of organometallic reagents to enones is a widely used process of carbon-carbon bond formation giving $\beta$-substituted carbonyl compounds which are versatile substrates used in further organic transformations. ${ }^{2}$ It is therefore of high value to achieve such a transformation in a catalytic and asymmetric fashion, which has been developed in the past few years using a large number of strategies. ${ }^{3}$

While transition-metal-catalyzed asymmetric transformations in the presence of chiral ligands have proved to be one of the most efficient methods for the construction of enantioenriched chiral compounds from achiral precursors, ${ }^{4}$ rhodium-catalyzed synthesis, including 1,4-conjugate addition of aryl- and alkenylboronic acids to enones, has experienced considerable growth over the past two decades. ${ }^{5}$

Indeed, a variety of chiral ligands in Rh-catalyzed asymmetric reactions have been used and developed including phosphorus, diene, and sulfoxide structures. ${ }^{6}$ In the last 10 years, chiral dienes ligands have emerged due to their efficiency in rhodiumcatalyzed 1,4-addition. These new ligands are superior to conventional chiral bis(phosphine) ligands in some cases, allowing the easier insertion of the boronic acid permitting a better control of regioselectivity. In particular, Hayashi et al. have developed a new class of ligands based on the bicyclo[2.2.2] octa-2,5-diene (bod) skeleton as good candidates for asymmetric 1,4-addition, for instance, on maleimide derivatives (Scheme 1, eq 1). ${ }^{7}$ Herein we report an uncommon rhodium-catalyzed asymmetric 1,4-addition of organoboronic
Scheme 1. Rh/bod-Catalyzed 1,4-Addition of Boron Species ref $7 \mathbf{i}$ (Duan et al. Tetrahedron 2007, 63, 8529)<smiles>O=C1C=CC(=O)N1[Al]</smiles>

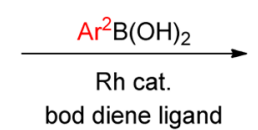<smiles>O=C1CC(=[Te])C(=O)N1[Al]</smiles>

ref 8 (Allen et al. Org. Biomol. Chem. 2012, 10, 32)<smiles>CC1(C)OC(=O)C(=C[Al])C(=O)O1</smiles>
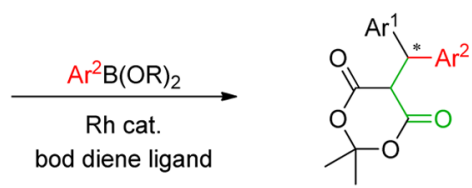

this work
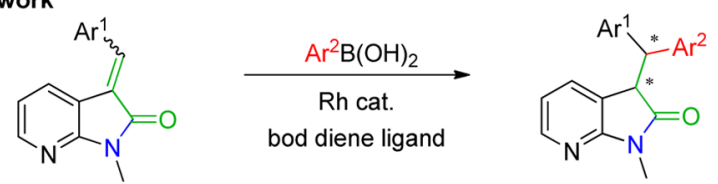

acids to 7-azaoxindoles (3-benzylidene-1H-pyrrolo[2,3-b]pyridine-2(3H)-ones) using a chiral diene ligand (Scheme 1 , eq 3). Indeed, as far as we know, it is the first time that this kind of reaction has been used on exocyclic methylene of lactam as Michael acceptor substrates. This 1,4-addition has been performed once toward exocyclic methylene on benzylidene Meldrum acid derivatives (Scheme 1, eq 2). ${ }^{8}$

We began our studies by synthesizing 3-benzylidene- $1 \mathrm{H}$ pyrrolo $[2,3-b]$ pyridin-2(3H)-ones by condensation between 7 azaoxindoles with various aldehydes (Table 1$){ }^{9}$

Compounds 1a-e have been obtained in a mixture of $E$ and $Z$ isomers with moderate to good yields. The NMR studies and comparisons with the literature have shown that the $E$ isomer is the major one. ${ }^{10}$ Several attempts have been made to separate

Received: December 9, 2014 
Table 1. Synthesis of 3-Benzylidene-1H-pyrrolo[2,3b] pyridin-2(3H)-ones<smiles>[R]N1C(=O)Cc2cccnc21</smiles>
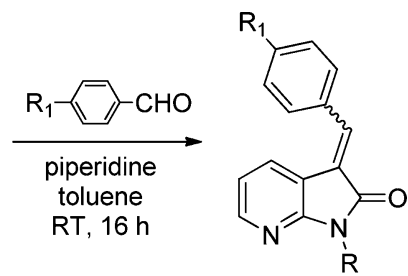

1a-e

\begin{tabular}{cllccc} 
entry & $\mathrm{R}$ & \multicolumn{1}{c}{$\mathrm{R}_{1}$} & product & yield $(\%)$ & $E / Z$ ratio $^{a}$ \\
\hline 1 & $\mathrm{Me}$ & $\mathrm{COMe}$ & $\mathbf{1 a}$ & 76 & $82 / 18$ \\
2 & $\mathrm{Me}$ & $\mathrm{H}$ & $\mathbf{1 b}$ & 85 & $94 / 6$ \\
3 & $\mathrm{Me}$ & $\mathrm{OMe}$ & $\mathbf{1 c}$ & 70 & $80 / 20$ \\
4 & $\mathrm{H}$ & $\mathrm{H}$ & $\mathbf{1 d}$ & 52 & $90 / 10$ \\
5 & $\mathrm{Bn}$ & $\mathrm{H}$ & $\mathbf{1 e}$ & 72 & $93 / 7$
\end{tabular}

${ }^{a}$ Determined by ${ }^{1} \mathrm{H}$ NMR analysis immediately after column chromatography.

isomers by classical chromatography and semipreparative chiral HPLC, but none of them was successful. Indeed, as an example, ${ }^{1} \mathrm{H}$ NMR and HPLC studies have shown that each isomer of $\mathbf{1 b}$ isomerizes in solution to an approximately $70 / 30(E / Z)$ ratio after 1 week. $^{11}$ However, 3-benzylidene- $1 H$-pyrrolo[2,3-b]pyridin-2(3H)-ones 1a-e did not isomerize when they were retained in the solid state.

The addition of phenylboronic acid to 3-benzylidene- $1 H$ pyrrolo[2,3- $b]$ pyridin-2(3H)-one derivatives $1 \mathbf{a}-\mathbf{e}$ was used to study the reaction (Table 2 ).

From previous unpublished biological results, the first stereoselective 1,4-addition reactions attempts were made on the 3-(4-acetoxybenzylidene)-1-methyl-1H-pyrrolo[2,3-b]pyridin-2(3H)-one 1a. In the presence of chlorobis(ethylene)rhodium(I) dimer and BINAP ligand, generally used in various organocatalysis combined with metal catalysis reactions, the 1,4-addition reaction on 1a did not occur, nor did it occur with the nonsubstituted $\mathbf{1 b}$ substrate (Table 2, entries 1-3). According to Hayashi's work, the use of $(R, R)$-Phbod* ligand gave better results. Indeed, 1,4-addition adducts starting from 1b, 1c, and 1e (Table 2, entries 5, 6, and 8) were obtained in good yields. However, these conditions have not been successful with 1a and 1d (Table 2, entries 4 and 7), so it seems that 1a is not a good Michael acceptor as it presents an electron-withdrawing group on the aromatic ring which may lead to a relocation of the double bond in the $\alpha, \beta$ positions of the amide to the aromatic ring. Finally, the addition was performed toward $\mathbf{1 b}$ and $\mathbf{1 e}$ with the nonchiral catalytic system $\left[(\mathrm{Rh}(\operatorname{cod}) \mathrm{Cl}]_{2}\right.$ and gave the adducts with modest yields (Table 2, entries 9 and 10).

Thereafter, to exemplify this reaction, these optimized experimental conditions with $(R, R)-\mathrm{Phbod}^{*}$ as the ligand and $\left[\left(\mathrm{RhCl}\left(\mathrm{C}_{2} \mathrm{H}_{4}\right)_{2}\right]_{2}\right.$ as the catalyst have been tested with various arylboronic acids toward compounds $\mathbf{1 b}$ and $\mathbf{1 e}$ as the Michael acceptor to furnish 1,4-adducts $\mathbf{2 a - j}$ (Table 3).

Employing the optimized set of reaction conditions with classical para- or meta-substituted arylboronic acids, the 1,4adducts $\mathbf{2} \mathbf{b}-\mathbf{f}$ were obtained with good yields (Table 3 , entries 2-6). In contrast, use of ortho-substituted arylboronic acids did not promote the reaction, and starting material was fully recovered (Table 3, entries 7 and 8 ). As seen before, the low reactivity of the boronic acids can be dependent on the steric hindrance. ${ }^{8}$

In order to obtain new interesting potential pharmaceutical derivatives, various heteroarylboronic acids have also been tested (Table 3, entries 9-13). Moreover, to the best of our knowledge, none of them have been described in rhodiumcatalyzed 1,4-addition. Several attempts have been done without success using 2-thiophenylboronic acid (Table 3, entry 10). In contrast, the reaction with the 3-substituted thiophene gave the adduct $2 \mathbf{h}$. This difference of reactivity is classical in heteroarylboronic acids. The lack of reactivity of the 2-pyridinylboronic acid was therefore expected, but surprisingly, no reaction occurred with 3-pyridinylboronic acid, whatever the quantity of rhodium catalyst used (Table 3, entries 12 and 13).

Then, starting from $\mathbf{1 e}$, we synthesized compounds $\mathbf{2 i}$ and $\mathbf{2 j}$, protected by a benzyl group with good yields (Table 3, entries 14 and 15). Indeed, as the nonprotected 7-azaoxindole derivative did not react (Table 1 ), we could use $N$-benzyl substrates $2 \mathbf{i}$ and $2 \mathbf{j}$ as precursors of unprotected adducts.

Table 2. Rhodium-Catalyzed 1,4-Addition of Phenylboronic Acid to 7-Azaoxindoles 1a-e: Study of Reaction Conditions

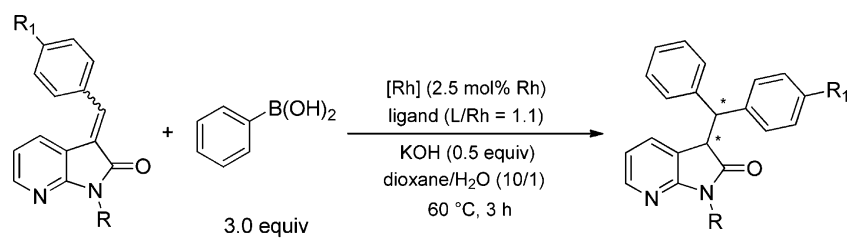

\begin{tabular}{|c|c|c|c|c|c|c|c|c|c|}
\hline Entry & 1a-e & {$[\mathrm{Rh}]$} & Ligand $^{[a]}$ & $\begin{array}{c}\text { Yield } \\
(\%)\end{array}$ & Entry & la-e & {$[\mathrm{Rh}]$} & Ligand $^{[a]}$ & $\begin{array}{c}\text { Yield } \\
(\%)\end{array}$ \\
\hline 1 & $1 \mathbf{a}$ & {$\left[\left(\mathrm{RhCl}\left(\mathrm{C}_{2} \mathrm{H}_{4}\right)_{2}\right]_{2}\right.$} & $(R)$-BINAP & 0 & 6 & $1 c$ & {$\left[\left(\mathrm{RhCl}\left(\mathrm{C}_{2} \mathrm{H}_{4}\right)_{2}\right]_{2}\right.$} & $(R, R)-$ Phbod* $^{*}$ & 85 \\
\hline 2 & $1 \mathbf{a}$ & {$\left[\left(\mathrm{RhCl}\left(\mathrm{C}_{2} \mathrm{H}_{4}\right)_{2}\right]_{2}{ }^{[\mathrm{b}]}\right.$} & (R)-BINAP & 0 & 7 & 1d & {$\left[\left(\mathrm{RhCl}\left(\mathrm{C}_{2} \mathrm{H}_{4}\right)_{2}\right]_{2}\right.$} & $(R, R)-$ Phbod $^{*}$ & 0 \\
\hline 3 & $1 b$ & {$\left[\left(\mathrm{RhCl}\left(\mathrm{C}_{2} \mathrm{H}_{4}\right)_{2}\right]_{2}\right.$} & $(R)$-BINAP & 0 & 8 & $1 e$ & {$\left[\left(\mathrm{RhCl}\left(\mathrm{C}_{2} \mathrm{H}_{4}\right)_{2}\right]_{2}\right.$} & $(R, R)-$ Phbod $^{*}$ & 90 \\
\hline 4 & $\mathbf{1 a}$ & {$\left[\left(\mathrm{RhCl}\left(\mathrm{C}_{2} \mathrm{H}_{4}\right)_{2}\right]_{2}\right.$} & $(R, R)-$ Phbod $^{*}$ & 0 & 9 & $\mathbf{1 b}$ & \multicolumn{2}{|c|}{$\left[(\mathrm{Rh}(\operatorname{cod}) \mathrm{Cl}]_{2}\right.$} & 45 \\
\hline 5 & $\mathbf{1 b}$ & {$\left[\left(\mathrm{RhCl}\left(\mathrm{C}_{2} \mathrm{H}_{4}\right)_{2}\right]_{2}\right.$} & $(R, R)-$ Phbod $^{*}$ & 93 & 10 & $1 \mathbf{e}$ & \multicolumn{2}{|c|}{$\left[(\mathrm{Rh}(\operatorname{cod}) \mathrm{Cl}]_{2}\right.$} & 48 \\
\hline [a] & & & & & & $\operatorname{cod})(\mathrm{Cl}]_{2}$ & [b] $10 \mathrm{~mol} \%$ in $\mathrm{Rh}$ & talyst were used & \\
\hline
\end{tabular}


Table 3. Rhodium-Catalyzed 1,4-Addition of Boronic Acids to 7-Azaoxindoles $1 \mathrm{~b}$ and $1 \mathrm{e}$<smiles>[R]N1C(=O)/C(=C/c2ccccc2)c2cccnc21</smiles>

$R=M e, 1 b$

$\mathrm{R}=\mathrm{Bn}, \mathbf{1 e}$

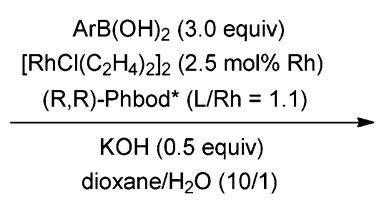

$60^{\circ} \mathrm{C}, 3 \mathrm{~h}$<smiles>[R]N1C(=O)c2cccnc2C([Al])c2ccccc21</smiles>

$2 a-j$

\begin{tabular}{|c|c|c|c|c|c|c|c|c|c|}
\hline Entry & $1 \mathrm{~b} / 1 \mathrm{e}$ & $\mathrm{Ar}$ & Product & $\begin{array}{c}\text { Yield } \\
(\%)\end{array}$ & Entry & $1 \mathrm{~b} / 1 \mathrm{e}$ & $\mathrm{Ar}$ & Product & $\begin{array}{c}\text { Yield } \\
(\%)\end{array}$ \\
\hline 1 & $1 b$ & & $2 a$ & 93 & 9 & $1 b$ & & $2 \mathrm{~g}$ & 86 \\
\hline 2 & $1 b$ & & $2 \mathbf{b}$ & 90 & 10 & $1 b$ & & - & n.r. ${ }^{[a]}$ \\
\hline 3 & $1 b$ & & $2 c$ & 86 & 11 & $1 b$ & & $2 \mathrm{~h}$ & $\begin{array}{l}35 \\
71^{[\mathrm{b}]}\end{array}$ \\
\hline 4 & $1 b$ & & $2 d$ & 89 & 12 & $1 b$ & & - & n.r. ${ }^{[a]}$ \\
\hline 5 & $1 b$ & & $2 e$ & 86 & 13 & $1 b$ & & - & n.r. ${ }^{[a]}$ \\
\hline 6 & $1 b$ & & $2 f$ & 73 & 14 & 1e & & $2 \mathrm{i}$ & 90 \\
\hline 7 & $1 b$ & & - & n.r. ${ }^{[a]}$ & 15 & $1 e$ & & $2 j$ & 90 \\
\hline 8 & $1 b$ & & - & n.r. ${ }^{[a]}$ & & & & & \\
\hline
\end{tabular}

As our optimized conditions used an asymmetric catalyst, we decided to study by chiral HPLC the reaction enantioselectivity on our best example in term of yield (Table 3, entry 2 ). The addition of 4-methoxyphenylboronic acid on 3-benzylidene-1methyl-1H-pyrrolo[2,3-b] pyridin-2(3H)-one $\mathbf{1 b}$ was considered, using either $\left[(\mathrm{Rh}(\operatorname{cod}) \mathrm{Cl}]_{2}\right.$ or $\left[\left(\mathrm{RhCl}\left(\mathrm{C}_{2} \mathrm{H}_{4}\right)_{2}\right]_{2} /(R, R)\right.$ Phbod* as the catalytic system (Scheme 2 ).

\section{Scheme 2. Reaction Enantioselectivity Study}
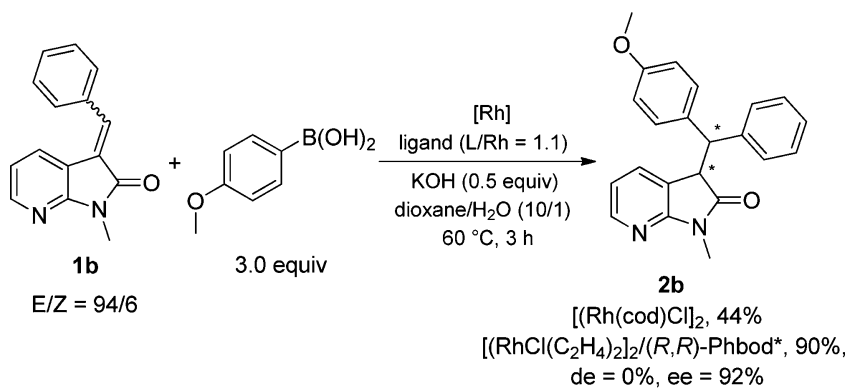

To begin, we optimized the separation of the four stereoisomers adducts obtained with the nonchiral addition using $\left[(\mathrm{Rh}(\operatorname{cod}) \mathrm{Cl}]_{2}\right.$ (Table 2 , entry 9$)$ in order to confirm the results of the chiral reaction (i.e., peak attribution). ${ }^{12}$ Unexpectedly, the enantioselective version using [( $\mathrm{RhCl}-$ $\left.\left(\mathrm{C}_{2} \mathrm{H}_{4}\right)_{2}\right]_{2} /(R, R)$-Phbod* ${ }^{*}$ gave only two isomers. ${ }^{13}$ This high enantioselectivity (ee $=92 \%$ ) showed us that the $E / Z$ isomer distribution in the starting material might not be responsible for the stereoisomer adduct distribution. This hypothesis was proven by performing the same addition with a different $E / Z$ ratio $(85 / 15)$ of the starting $\mathbf{1 b}$, yielding the two isomers in the same enantiomeric ratio. ${ }^{14}$ It seems that a dynamic resolution or an enantioconvergent process operates and provides high enantioselectivity whatever the $E / Z$ ratio of the starting material. There is no diastereomeric excess, which is not surprising considering that the protonation of $\mathrm{Rh}$-enolates in the Hayashi reaction is often nonselective. Furthermore, the acidity of the C-3 hydrogen is likely to lead to epimerization under the reaction conditions.

Finally, we have extended this study to compounds described in Table 3. The results are mentioned in Figure 1.

In summary, we have identified a novel effective and highly stereoselective approach for the preparation of original 7azaoxindole derivatives using a rhodium-catalyzed asymmetric 1,4-addition of various arylboronic acids toward 3-benzylidene$1 H$-pyrrolo[2,3-b]pyridin-2(3H)-ones.

\section{EXPERIMENTAL SECTION}

General Considerations. Unless otherwise noted, all reagents were purchased from commercial suppliers and used without purification. All 1,4-addition reactions were performed under inert atmosphere. Silica gel (230-400 mesh) was used for column chromatography. ${ }^{1} \mathrm{H}$ NMR spectra were referenced internally to the residual proton resonance in $\mathrm{CDCl}_{3}(\delta 7.26 \mathrm{ppm})$ or with TMS $(\delta$ $0.00 \mathrm{ppm})$ as the internal standard. Chemical shifts $(\delta)$ were reported as parts per million (ppm) on the $\delta$ scale downfield from TMS. ${ }^{13} \mathrm{C}$ NMR spectra were referenced to $\mathrm{CDCl}_{3}(\delta 77.0 \mathrm{ppm}$, the middle peak). Coupling constants $(J)$ are reported in hertz.

High-resolution mass spectra (HRMS) were performed using Orbitrap. HPLC analysis were carried out with a LaChrom Elite system using $25 \mathrm{~nm}$ UV detection to quantify diastereoisomers.

General Procedure for the Condensation of Aldehyde on 7Azaoxindole. A solution of the $1 H$-pyrrolo[2,3-b]pyridin-2(3H)-one $(6.75 \mathrm{mmol})$, the appropriate aldehyde $(13.5 \mathrm{mmol})$, and piperidine $(2.7 \mathrm{mmol})$ in toluene $(15 \mathrm{~mL})$ under an $\mathrm{Ar}$ atmosphere was stirred at ambient temperature for $16 \mathrm{~h}$. The reaction mixture was treated with $\mathrm{H}_{2} \mathrm{O}$, and the aqueous layer was extracted with EtOAc. The organic layers were dried over $\mathrm{MgSO}_{4}$, filtered, concentrated in vacuo, and purified by flash chromatography $\left(\mathrm{SiO}_{2}, \mathrm{DCM} / \mathrm{AcOEt}\right)$ to afford the desired 3-benzylidene-1H-pyrrolo[2,3-b] pyridin-2(3H)-one derivative.

3-(4-Acetylbenzylidene)-1-methyl-1H-pyrrolo[2,3-b]pyridin2(3H)-one (1a): yellow solid; $1.43 \mathrm{~g}(76 \%) ; \mathrm{mp} 152-153{ }^{\circ} \mathrm{C} ;{ }^{1} \mathrm{H}$ NMR $\left(\mathrm{CDCl}_{3}\right) \delta 8.17(\mathrm{dd}, J=5.2,1.5 \mathrm{~Hz}, 1 \mathrm{H}), 8.07-8.02(\mathrm{~m}, 2 \mathrm{H})$, $7.93(\mathrm{~s}, 1 \mathrm{H}), 7.72-7.69(\mathrm{~m}, 3 \mathrm{H}), 6.81$ (dd, $J=7.6,5.3 \mathrm{~Hz}, 1 \mathrm{H}), 3.36$ $(\mathrm{s}, 3 \mathrm{H}), 2.65(\mathrm{~s}, 3 \mathrm{H}) ;{ }^{13} \mathrm{C} \mathrm{NMR}\left(\mathrm{CDCl}_{3}\right) \delta 197.3,167.8,157.4,148.4$, 
<smiles>CN1C=Nc2ccc(c(-c3ccc(C=O)cc3)c2-c2ccccc2)C1=O</smiles>

$\left[(\mathrm{Rh}(\operatorname{cod}) \mathrm{Cl}]_{2}, 31 \%\right.$

$\left[\left(\mathrm{RhCl}\left(\mathrm{C}_{2} \mathrm{H}_{4}\right)_{2}\right]_{2} /(R, R)-\mathrm{Phbod}^{*}, 86 \%\right.$, de $=0 \%$, ee $=92 \%$

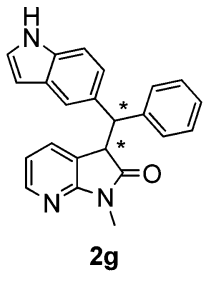

$\left[(\mathrm{Rh}(\operatorname{cod}) \mathrm{Cl}]_{2}, 40 \%\right.$

$\left[\left(\mathrm{RhCl}\left(\mathrm{C}_{2} \mathrm{H}_{4}\right)_{2}\right]_{2} /(R, R)-\mathrm{Phbod}^{*}, 86 \%\right.$ de $=0 \%$, ee $=94 \%$<smiles>Cc1ccc(C(=C2C(=O)N(c3ccccc3)c3ncccc32)c2ccccc2)cc1</smiles>

$\left[(\mathrm{Rh}(\operatorname{cod}) \mathrm{Cl}]_{2}, 36 \%\right.$

$\left[\left(\mathrm{RhCl}\left(\mathrm{C}_{2} \mathrm{H}_{4}\right)_{2}\right]_{2} /(R, R)-\mathrm{Phbod}^{*}, 89 \%\right.$, de $=0 \%$, ee $>80 \%$

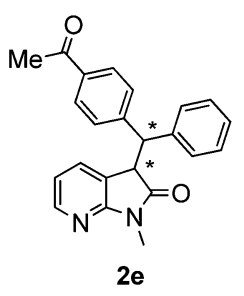

$\left[(\mathrm{Rh}(\mathrm{cod}) \mathrm{Cl}]_{2}, 50 \%\right.$

$\left[\left(\mathrm{RhCl}\left(\mathrm{C}_{2} \mathrm{H}_{4}\right)_{2}\right]_{2} /(R, R)-\mathrm{Phbod}^{*}, 86 \%\right.$ $\mathrm{de}=0 \%$, ee $=96 \%$

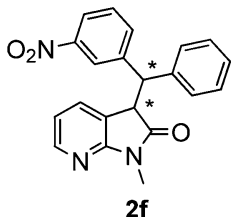

$\left[(\mathrm{Rh}(\operatorname{cod}) \mathrm{Cl}]_{2}\right.$, no reaction $\left[\left(\mathrm{RhCl}\left(\mathrm{C}_{2} \mathrm{H}_{4}\right)_{2}\right]_{2} /(R, R)-\right.$ Phbod $^{*}, 73 \%$, $\mathrm{de}=0 \%$, ee $=$ n.d. ${ }^{*}$

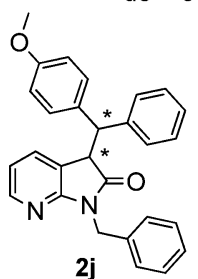

$\left[\left(\mathrm{Rh}(\mathrm{cod}) \mathrm{Cl}_{2}, 40 \%\right.\right.$

$\left[(\mathrm{Rh}(\mathrm{cod}) \mathrm{Cl}]_{2}\right.$, no reaction $\left[\left(\mathrm{RhCl}\left(\mathrm{C}_{2} \mathrm{H}_{4}\right)_{2}\right]_{2} /(R, R)-\mathrm{Phbod}^{*}, 71 \%\right.$, de $=0 \%$, ee $=$ n.d. ${ }^{*}$
$\left[\left(\mathrm{RhCl}\left(\mathrm{C}_{2} \mathrm{H}_{4}\right)_{2}\right]_{2} /(R, R)-\mathrm{Phbod}^{*}, 90 \%\right.$, de $=0 \%$, ee $=88 \%$

*n.d.: non determined ${ }^{15}$

Figure 1. de and ee values for compounds $2 \mathrm{c}-\mathbf{h}$ and $\mathbf{2 j}$.

147.6, 139.2, 137.9, 137.3, 137.2, 132.1, 129.8, 129.6, 128.9, 128.3, 117.7, 26.8, 25.6; HRMS (ESI) $m / z$ calcd for $\mathrm{C}_{17} \mathrm{H}_{15} \mathrm{~N}_{2} \mathrm{O}_{2}(\mathrm{M}+\mathrm{H})$ 279.11280, found 279.11246

3-Benzylidene-1-methyl-1H-pyrrolo[2,3-b]pyridin-2(3H)-one (1b): yellow solid. $1.35 \mathrm{~g}(85 \%) ; \mathrm{mp} 98-99{ }^{\circ} \mathrm{C}$; ${ }^{1} \mathrm{H}$ NMR $\left(\mathrm{CDCl}_{3}\right) \delta 8.15$ (dd, $J=5.3,1.5 \mathrm{~Hz}, 1 \mathrm{H}), 7.96(\mathrm{~s}, 1 \mathrm{H}), 7.82(\mathrm{dd}, J=7.5,1.3 \mathrm{~Hz}, 1 \mathrm{H})$, 7.62-7.59 (m, 2H), 7.51-7.44 (m, 3H), $6.81(\mathrm{dd} J=7.6,5.3 \mathrm{~Hz}$, $1 \mathrm{H}), 3.37$ (s, $3 \mathrm{H}) ;{ }^{13} \mathrm{C} \mathrm{NMR}\left(\mathrm{CDCl}_{3}\right)$ 168.2, 157.0, 147.7, 139.3, 134.5, 130.2, 129.6, 129.5, 128.9, 125.5, 117.6, 116.1, 25.5; HRMS (ESI) $\mathrm{m} / z$ calcd for $\mathrm{C}_{15} \mathrm{H}_{13} \mathrm{~N}_{2} \mathrm{O}(\mathrm{M}+\mathrm{H})$ 237.10224, found 237.10143.

3-(4-Methoxybenzylidene)-1-methyl-1H-pyrrolo[2,3-b]pyridin2(3H)-one (1c): yellow solid; $1.26 \mathrm{~g}(70 \%) ; \mathrm{mp} 114-116{ }^{\circ} \mathrm{C} ;{ }^{1} \mathrm{H}$ $\operatorname{NMR}\left(\mathrm{CDCl}_{3}\right) \delta 8.16(\mathrm{dd}, J=5.2,1.5 \mathrm{~Hz}, 1 \mathrm{H}), 7.95-7.92(\mathrm{~m}, 2 \mathrm{H})$, 7.63-7.60 (m, 2H), 7.03-6.97 (m, 2H), $6.84(\mathrm{dd}, J=7.5,5.2 \mathrm{~Hz}$, $1 \mathrm{H}), 3.89$ (s, 3H), $3.38(\mathrm{~s}, 3 \mathrm{H}) ;{ }^{13} \mathrm{C}$ NMR $\left(\mathrm{CDCl}_{3}\right) \delta 168.5,161.4$, $156.7,147.1,139.5,135.1,131.8,129.1,123.3,117.5,114.4,114.1$, 55.6, 25.6; HRMS (ESI) $m / z$ calcd for $\mathrm{C}_{16} \mathrm{H}_{15} \mathrm{~N}_{2} \mathrm{O}_{2}(\mathrm{M}+\mathrm{H}$ ) 267.11280, found 267.11176 .

3-Benzylidene-1H-pyrrolo[2,3-b]pyridin-2(3H)-one (1d): yellow solid; $0.78 \mathrm{~g}$ (52\%); mp 166-168 ${ }^{\circ} \mathrm{C}$; ${ }^{1} \mathrm{H}$ NMR (DMSO) $\delta 11.26$ $(\mathrm{s}, 1 \mathrm{H}), 8.10(\mathrm{~d}, J=5.1 \mathrm{~Hz}, 1 \mathrm{H}), 7.82(\mathrm{~d}, J=7.6 \mathrm{~Hz}, 1 \mathrm{H}), 7.77(\mathrm{~s}$, $1 \mathrm{H}), 7.72(\mathrm{~d}, J=7.3 \mathrm{~Hz}, 2 \mathrm{H}), 7.52(\mathrm{~m}, 3 \mathrm{H}), 6.95-6.86(\mathrm{~m}, 1 \mathrm{H}) ;{ }^{13} \mathrm{C}$ NMR (DMSO) $\delta 168.2,157.0,148.2,137.8,134.0,130.2,129.6$, $128.9,128.3,126.0,117.4,115.3$; HRMS (ESI) $\mathrm{m} / \mathrm{z}$ calcd for $\mathrm{C}_{14} \mathrm{H}_{11} \mathrm{~N}_{2} \mathrm{O}(\mathrm{M}+\mathrm{H})$ 223.08659, found 223.08614.

1-Benzyl-3-benzylidene-1H-pyrrolo[2,3-b]pyridin-2(3H)-one (1e): yellow solid; $1.51 \mathrm{~g}(72 \%)$; mp $118-120{ }^{\circ} \mathrm{C} ;{ }^{1} \mathrm{H}$ NMR $\left(\mathrm{CDCl}_{3}\right) \delta$ $8.18(\mathrm{dd}, J=5.2,1.5 \mathrm{~Hz}, 1 \mathrm{H}), 8.00(\mathrm{~s}, 1 \mathrm{H}), 7.85(\mathrm{dd}, J=7.6,1.4 \mathrm{~Hz}$, $1 \mathrm{H}), 7.65-7.62(\mathrm{~m}, 2 \mathrm{H}), 7.53-7.49(\mathrm{~m}, 5 \mathrm{H}), 7.32(\mathrm{ddt}, J=7.0,5.5$, $2.2 \mathrm{~Hz}, 3 \mathrm{H}), 6.83(\mathrm{dd}, J=7.6,5.2 \mathrm{~Hz}, 1 \mathrm{H}), 5.12(\mathrm{~s}, 2 \mathrm{H}) ;{ }^{13} \mathrm{C} \mathrm{NMR}$ $\left(\mathrm{CDCl}_{3}\right) \delta 168.1,156.6,148.1,139.3,136.9,134.6,132.3,130.2,129.6$, $129.5,129.0,128.7,128.6,127.7,125.6,117.7,42.8$. HRMS (ESI) $\mathrm{m} / z$ calcd for $\mathrm{C}_{21} \mathrm{H}_{17} \mathrm{~N}_{2} \mathrm{O}(\mathrm{M}+\mathrm{H})$ 313.13354, found 313.13272.

General Procedure for the $\mathrm{Rh}(\mathrm{I})$-Catalyzed 1,4-Conjugate Addition of Arylboronic Acid to 7-Azaoxindole. A solution of $\left[\mathrm{RhCl}\left(\mathrm{C}_{2} \mathrm{H}_{4}\right)_{2}\right]_{2}(8 \mathrm{mg}, 0,04 \mathrm{mmol} \mathrm{Rh})$ and $(R, R)$-Ph-bod* $(12 \mathrm{mg}$, $0,046 \mathrm{mmol}$ ) in $2 \mathrm{~mL}$ of 1,4-dioxane was stirred for $10 \mathrm{~min}$ at room temperature under Ar. A $0.21 \mathrm{~mL}$ portion of a $1 \mathrm{M} \mathrm{KOH}$ solution $(0,21 \mathrm{mmol})$ was then added, and the resulting solution was stirred for $3 \mathrm{~min}$ at room temperature. After addition of $\mathrm{ArB}(\mathrm{OH})_{2}(1,27 \mathrm{mmol})$, the mixture was stirred for an additional $3 \mathrm{~min}$ at room temperature. The substrate 1, 3-benzylidene-1-methyl-1H-pyrrolo[2,3-b]pyridin-
2(3H)-one (100 mg, $0.42 \mathrm{mmol}$ ), was then added to this with additional $2 \mathrm{~mL}$ of 1,4-dioxane, and the resulting mixture was stirred for $3 \mathrm{~h}$ at $60{ }^{\circ} \mathrm{C}$. The solvent was removed under vacuum, and the resulting mixture was purified by silica gel flash chromatography with cyclohexane/EtOAc to afford the desired 1,4-adduct.

3-Benzhydryl-1-methyl-1H-pyrrolo[2,3-b]pyridin-2(3H)-one (2a): oil; $122 \mathrm{mg}$ (93\%); ${ }^{1} \mathrm{H}$ NMR $\left(\mathrm{CDCl}_{3}\right) \delta 8.12$ (ddd, $J=5.2,1.6,0.8$ $\mathrm{Hz}, 1 \mathrm{H}), 7.38-7.27(\mathrm{~m}, 5 \mathrm{H}), 7.19-7.14(\mathrm{~m}, 3 \mathrm{H}), 7.01-6.95(\mathrm{~m}, 2 \mathrm{H})$, 6.89-6.84 (m, 1H), $6.77(\mathrm{dd}, J=7.3,5.2 \mathrm{~Hz}, 1 \mathrm{H}), 4.91(\mathrm{~d}, J=5.7 \mathrm{~Hz}$, $1 \mathrm{H}), 4.37(\mathrm{~d}, J=5.7 \mathrm{~Hz}, 1 \mathrm{H}), 3.08(\mathrm{~s}, 3 \mathrm{H}) ;{ }^{13} \mathrm{C} \mathrm{NMR}\left(\mathrm{CDCl}_{3}\right) \delta$ $175.7,157.8,147.1,141.0,139.4,132.5,129.3,128.6,128.4,128.4$, 127.3, 127.0, 122.0, 117.7, 51.5, 49.5, 25.2; HRMS (ESI) $\mathrm{m} / z$ calcd for $\mathrm{C}_{21} \mathrm{H}_{19} \mathrm{~N}_{2} \mathrm{O}(\mathrm{M}+\mathrm{H})$ 315.14919, found 315.14816.

3-((4-Methoxyphenyl)(phenyl)methyl)-1-methyl-1H-pyrrolo[2,3b]pyridin-2(3H)-one (2b): oil; $130 \mathrm{mg}(90 \%) ;{ }^{1} \mathrm{H}$ NMR $\left(\mathrm{CDCl}_{3}\right) \delta$ $8.14(\mathrm{dd}, J=5.3,0.8 \mathrm{~Hz}, 2 \mathrm{H}), 7.41-7.26(\mathrm{~m}, 5 \mathrm{H}), 7.26-7.14(\mathrm{~m}$, $5 \mathrm{H}), 7.05-6.66(\mathrm{~m}, 12 \mathrm{H}), 4.91(\mathrm{~d}, J=5.5 \mathrm{~Hz}, 1 \mathrm{H}), 4.86(\mathrm{~d}, J=5.8$ $\mathrm{Hz}, 1 \mathrm{H}), 4.36(\mathrm{t}, J=5.6 \mathrm{~Hz}, 2 \mathrm{H}), 3.83(\mathrm{~s}, 3 \mathrm{H}), 3.75(\mathrm{~s}, 3 \mathrm{H}), 3.13(\mathrm{~s}$, $3 \mathrm{H}), 3.12(\mathrm{~s}, 3 \mathrm{H}) ;{ }^{13} \mathrm{C} \mathrm{NMR}\left(\mathrm{CDCl}_{3}\right) \delta 175.7,158.7,146.4,141.4$, $139.7,132.9,131.2,130.4,129.5,129.2,128.7,128.4,128.4,127.3$, 127.0, 122.4, 117.7, 114.0, 113.8, 55.4, 55.27, 50.9, 50.6, 49.7, 25.5; HRMS (ESI) $m / z$ calcd for $\mathrm{C}_{22} \mathrm{H}_{21} \mathrm{~N}_{2} \mathrm{O}_{2}(\mathrm{M}+\mathrm{H}), 345.15975$, found 345.15878 .

4-((1-Methyl-2-oxo-2,3-dihydro-1H-pyrrolo[2,3-b]pyridin-3-yl)(phenyl)methyl)benzaldehyde (2c): oil; $122 \mathrm{mg}(86 \%) ;{ }^{1} \mathrm{H}$ NMR $\left(\mathrm{CDCl}_{3}\right): \delta 10.02(\mathrm{~s}, 1 \mathrm{H}), 9.94(\mathrm{~s}, 1 \mathrm{H}), 8.18-8.13(\mathrm{~m}, 2 \mathrm{H}), 7.88(\mathrm{~d}, J$ $=8.3 \mathrm{~Hz}, 2 \mathrm{H}), 7.74-7.68(\mathrm{~m}, 2 \mathrm{H}), 7.52(\mathrm{~d}, J=8.2 \mathrm{~Hz}, 2 \mathrm{H}), 7.40-$ $7.16(\mathrm{~m}, 10 \mathrm{H}), 7.12-6.97(\mathrm{~m}, 3 \mathrm{H}), 6.81(\mathrm{~m}, 3 \mathrm{H}), 5.07(\mathrm{~d}, J=5.1 \mathrm{~Hz}$, $1 \mathrm{H}), 4.82(\mathrm{~d}, J=6.6 \mathrm{~Hz}, 1 \mathrm{H}), 4.43(\mathrm{~d}, J=5.8 \mathrm{~Hz}, 2 \mathrm{H}), 3.13(\mathrm{~s}, 3 \mathrm{H})$, $3.11(\mathrm{~s}, 3 \mathrm{H}) ;{ }^{13} \mathrm{C} \mathrm{NMR}\left(\mathrm{CDCl}_{3}\right) \delta 191.9,191.9,175.3,175.1,157.7$, $148.3,147.3,147.2,146.6,139.9,139.0,135.4,135.3,132.6,132.5$, $130.1,130.0,129.8,129.2,128.9,128.8,128.4,127.8,127.5,121.7$, 121.4, 117.9, 117.8, 52.2, 51.4, 49.2, 48.9, 25.4; HRMS (ESI) $\mathrm{m} / z$ calcd for $\mathrm{C}_{22} \mathrm{H}_{19} \mathrm{~N}_{2} \mathrm{O}_{2}(\mathrm{M}+\mathrm{H})$ 343.14410, found 343.14325.

1-Methyl-3-(phenyl(p-tolyl)methyl)-1H-pyrrolo[2,3-b]pyridin2(3H)-one (2d): oil; $122 \mathrm{mg}(89 \%) ;{ }^{1} \mathrm{H} \mathrm{NMR}\left(\mathrm{CDCl}_{3}\right) \delta 8.14$ (d, $J=$ $5.2 \mathrm{~Hz}, 2 \mathrm{H}), 7.42-7.27(\mathrm{~m}, 6 \mathrm{H}), 7.24-7.14(\mathrm{~m}, 6 \mathrm{H}), 7.02-6.96(\mathrm{~m}$, $4 \mathrm{H}), 6.89$ (ddd, $J=11.0,5.3,3.7 \mathrm{~Hz}, 4 \mathrm{H}), 6.79$ (dd, $J=7.3,5.3 \mathrm{~Hz}$, $2 \mathrm{H}), 4.89(\mathrm{t}, J=6.1 \mathrm{~Hz}, 2 \mathrm{H}), 4.37(\mathrm{~d}, J=5.4 \mathrm{~Hz}, 2 \mathrm{H}), 3.12(\mathrm{~s}, 3 \mathrm{H})$, $3.11(\mathrm{~s}, 3 \mathrm{H}), 2.37(\mathrm{~s}, 3 \mathrm{H}), 2.27(\mathrm{~s}, 3 \mathrm{H}) ;{ }^{13} \mathrm{C} \mathrm{NMR}(\mathrm{CDCl} 3) \delta 175.7$, $157.7,146.6,141.3,139.6,138.0,136.9,136.7,136.2,132.8,129.3$, $129.3,129.2,129.1,128.6,128.4,128.4,128.3,127.3,127.0,122.3$, 
117.7, 51.2, 51.1, 49.6, 49.5, 25.4, 21.1; HRMS (ESI) $m / z$ calcd for $\mathrm{C}_{22} \mathrm{H}_{21} \mathrm{~N}_{2} \mathrm{O}(\mathrm{M}+\mathrm{H})$ 329.16484, found 329.16397.

3-((4-Acetylphenyl)(phenyl)methyl)-1-methyl-1H-pyrrolo[2,3-b]pyridin-2(3H)-one (2e): oil; $127 \mathrm{mg}(86 \%) ;{ }^{1} \mathrm{H} \mathrm{NMR}\left(\mathrm{CDCl}_{3}\right) \delta 8.13$ $(\mathrm{d}, J=4.9 \mathrm{~Hz}, 2 \mathrm{H}), 7.93(\mathrm{~d}, J=8.4 \mathrm{~Hz}, 2 \mathrm{H}), 7.81-7.70(\mathrm{~m}, 2 \mathrm{H})$, $7.47-7.15(\mathrm{~m}, 10 \mathrm{H}), 7.11(\mathrm{t}, J=8.9 \mathrm{~Hz}, 2 \mathrm{H}), 7.07-6.98(\mathrm{~m}, 2 \mathrm{H})$, $6.94(\mathrm{t}, J=8.3 \mathrm{~Hz}, 1 \mathrm{H}), 6.78(\mathrm{ddd}, J=10.1,7.3,5.3 \mathrm{~Hz}, 3 \mathrm{H}), 5.02(\mathrm{~d}$, $J=5.2 \mathrm{~Hz}, 1 \mathrm{H}), 4.81(\mathrm{~d}, J=6.4 \mathrm{~Hz}, 1 \mathrm{H}), 4.39(\mathrm{~d}, J=5.9 \mathrm{~Hz}, 2 \mathrm{H})$, $3.10(\mathrm{~s}, 3 \mathrm{H}) 3.09(\mathrm{~s}, 3 \mathrm{H}), 2.59(\mathrm{~s}, 3 \mathrm{H}), 2.53(\mathrm{~s}, 3 \mathrm{H}) ;{ }^{13} \mathrm{C}$ NMR $\left(\mathrm{CDCl}_{3}\right): \delta 197.8,197.7,175.3,175.2,157.6,147.1,147.0,146.6$, $145.0,140.1,139.0,136.1,135.9,132.7,132.6,129.6,129.2,128.8$, $128.8,128.7,128.6,128.5,128.4,127.7,127.4,121.8,121.6,117.9$, $117.8,51.9,51.2,49.3,49.0,26.7,26.7,25.5$; HRMS (ESI) $\mathrm{m} / z$ calcd for $\mathrm{C}_{23} \mathrm{H}_{21} \mathrm{~N}_{2} \mathrm{O}_{2}(\mathrm{M}+\mathrm{H})$ 357.15975, found 357.15869.

1-Methyl-3-((3-nitrophenyl)(phenyl)methyl)-1H-pyrrolo[2,3-b]pyridin-2(3H)-one (2f): oil; $109 \mathrm{mg}(73 \%) ;{ }^{1} \mathrm{H}$ NMR $(300 \mathrm{MHz}$, $\left.\mathrm{CDCl}_{3}\right) \delta 8.25(\mathrm{~s}, 1 \mathrm{H}), 8.14(\mathrm{dd}, J=6.3,3.5 \mathrm{~Hz}, 3 \mathrm{H}), 8.11-8.00(\mathrm{~m}$, $1 \mathrm{H}), 7.87(\mathrm{t}, J=1.8 \mathrm{~Hz}, 1 \mathrm{H}), 7.70(\mathrm{~d}, J=7.8 \mathrm{~Hz}, 1 \mathrm{H}), 7.53(\mathrm{t}, J=7.9$ $\mathrm{Hz}, 1 \mathrm{H}), 7.47-7.18(\mathrm{~m}, 10 \mathrm{H}), 7.12(\mathrm{dd}, J=9.0,5.5 \mathrm{~Hz}, 3 \mathrm{H}), 6.91-$ $6.68(\mathrm{~m}, 3 \mathrm{H}), 5.12(\mathrm{~d}, J=4.6 \mathrm{~Hz}, 1 \mathrm{H}), 4.72(\mathrm{~d}, J=7.3 \mathrm{~Hz}, 1 \mathrm{H}), 4.43$ $(\mathrm{d}, J=5.2 \mathrm{~Hz}, 2 \mathrm{H}), 3.14(\mathrm{~s}, 3 \mathrm{H}), 3.10(\mathrm{~s}, 3 \mathrm{H}) ;{ }^{13} \mathrm{C} \mathrm{NMR}(75 \mathrm{MHz}$, $\left.\mathrm{CDCl}_{3}\right) \delta 175.2$, 174.9, 157.6, 148.4, 148.1, 147.5, 147.4, 143.5, 141.7, $139.4,139.1,135.6,134.7,132.6,132.5,129.5,129.3,129.0,129.0$, $128.5,128.0,127.7,124.2,123.2,122.4,122.2,121.4,121.0,118.0$, 117.9, 52.0, 50.9, 49.2, 48.6, 25.4, 25.4; HRMS (ESI) $\mathrm{m} / z$ calcd for $\mathrm{C}_{21} \mathrm{H}_{18} \mathrm{~N}_{3} \mathrm{O}_{3}(\mathrm{M}+\mathrm{H})$ 360.13427, found 360.13358 .

3-((1H-Indol-5-yl)(phenyl)methyl)-1-methyl-1H-pyrrolo[2,3-b]pyridin-2(3H)-one (2g): oil; $127 \mathrm{mg}(86 \%) ;{ }^{1} \mathrm{H} \mathrm{NMR}\left(\mathrm{CDCl}_{3}\right) \delta 8.35$ (s, 1H), $8.24(\mathrm{~s}, 1 \mathrm{H}), 8.13$ (ddd, J = 5.2, 1.5, $0.7 \mathrm{~Hz}, 2 \mathrm{H}), 7.54$ (d, J = $0.8 \mathrm{~Hz}, 1 \mathrm{H}), 7.43-7.31(\mathrm{~m}, 6 \mathrm{H}), 7.31-7.26(\mathrm{~m}, 1 \mathrm{H}), 7.24-7.21(\mathrm{~m}$, $1 \mathrm{H}), 7.20-7.13(\mathrm{~m}, 6 \mathrm{H}), 7.06-6.97(\mathrm{~m}, 2 \mathrm{H}), 6.97-6.87(\mathrm{~m}, 1 \mathrm{H})$, $6.84-6.71(\mathrm{~m}, 3 \mathrm{H}), 6.51(\mathrm{ddd}, \mathrm{J}=3.0,2.0,0.9 \mathrm{~Hz}, 1 \mathrm{H}), 6.42(\mathrm{ddd}, \mathrm{J}=$ 3.0, 2.0, $0.9 \mathrm{~Hz}, 1 \mathrm{H}), 5.11(\mathrm{~d}, \mathrm{~J}=5.4 \mathrm{~Hz}, 1 \mathrm{H}), 4.99(\mathrm{~d}, \mathrm{~J}=6.0 \mathrm{~Hz}$, $1 \mathrm{H}), 4.51-4.42(\mathrm{~m}, 2 \mathrm{H}), 3.11(\mathrm{~s}, 3 \mathrm{H}), 3.10(\mathrm{~s}, 3 \mathrm{H}) ;{ }^{13} \mathrm{C}$ NMR $(75$ $\mathrm{MHz}) \delta 176.1,176.0,157.9,156.0,146.8,146.8,142.2,140.2,135.0$, $134.8,132.9,132.8,132.7,132.4,130.9,129.4,128.5,128.5,128.3$, $128.0,127.9,127.1,126.8,124.9,124.6,123.5,122.9,122.6,122.4$, 121.4, 120.4, 119.7, 117.7, 111.3, 110.9, 102.9, 102.8, 51.8, 51.4, 50.1, 49.8, 25.3, 25.3; HRMS (ESI) $\mathrm{m} / z$ calcd for $\mathrm{C}_{23} \mathrm{H}_{20} \mathrm{~N}_{3} \mathrm{O}(\mathrm{M}+\mathrm{H})$ 355.16344 , found 355.15955 .

1-Methyl-3-(phenyl(thiophene-3-yl)methyl)-1H-pyrrolo[2,3-b]pyridin-2(3H)-one (2h): oil; $95 \mathrm{mg}(71 \%) ;{ }^{1} \mathrm{H}$ NMR $\left(\mathrm{CDCl}_{3}\right) \delta 8.20-$ $8.11(\mathrm{~m}, 2 \mathrm{H}), 7.43-7.23(\mathrm{~m}, 5 \mathrm{H}), 7.20-7.09(\mathrm{~m}, 5 \mathrm{H}), 7.05-6.91(\mathrm{~m}$, $4 \mathrm{H}), 6.90-6.78(\mathrm{~m}, 3 \mathrm{H}), 6.57(\mathrm{dd}, J=5.0,1.3 \mathrm{~Hz}, 1 \mathrm{H}), 5.14(\mathrm{~d}, J=$ $4.9 \mathrm{~Hz}, 1 \mathrm{H}), 4.96(\mathrm{~d}, J=4.2 \mathrm{~Hz}, 1 \mathrm{H}), 4.32(\mathrm{~d}, J=4.9 \mathrm{~Hz}, 1 \mathrm{H}), 4.25$ $(\mathrm{d}, J=5.0 \mathrm{~Hz}, 1 \mathrm{H}), 3.12(\mathrm{~s}, 3 \mathrm{H}), 3.06(\mathrm{~s}, 3 \mathrm{H}) ;{ }^{13} \mathrm{C} \mathrm{NMR}\left(\mathrm{CDCl}_{3}\right) \delta$ $175.5,175.3,157.8,157.7,146.7,146.5,142.2,140.6,138.9,138.5$, $132.8,132.6,129.1,128.7,128.3,128.3,128.3,127.9,127.6,127.2$, $126.5,125.6,123.2,122.6,122.2,121.9,117.8,50.3,50.2,47.7,46.8$, 25.5, 25.4; HRMS (ESI) $m / z$ calcd for $\mathrm{C}_{19} \mathrm{H}_{17} \mathrm{~N}_{2} \mathrm{OS}(\mathrm{M}+\mathrm{H})$ 321.10561, found 321.10538 .

3-Benzhydryl-1-benzyl-1H-pyrrolo[2,3-b]pyridin-2(3H)-one (2i): oil; $143 \mathrm{mg}(90 \%) ;{ }^{1} \mathrm{H} \mathrm{NMR}\left(\mathrm{CDCl}_{3}\right): \delta 8.14$ (ddd, $J=5.2,1.5$, $0.8 \mathrm{~Hz}, 1 \mathrm{H}), 7.41-7.27(\mathrm{~m}, 5 \mathrm{H}), 7.24-7.10(\mathrm{~m}, 6 \mathrm{H}), 7.06-6.95(\mathrm{~m}$, $5 \mathrm{H}), 6.80(\mathrm{dd}, J=7.3,5.3 \mathrm{~Hz}, 1 \mathrm{H}), 5.04(\mathrm{~d}, J=5.2 \mathrm{~Hz}, 1 \mathrm{H}), 4.97$ (d, $J$ $=14.9 \mathrm{~Hz}, 1 \mathrm{H}), 4.79(\mathrm{~d}, J=14.9 \mathrm{~Hz}, 1 \mathrm{H}), 4.46(\mathrm{~d}, J=5.2 \mathrm{~Hz}, 1 \mathrm{H})$; ${ }^{13} \mathrm{C} \mathrm{NMR}\left(\mathrm{CDCl}_{3}\right) \delta 175.4,157.3,147.3,141.0,139.0,136.2,132.5$, 129.6, 128.4, 127.7, 127.3, 127.2, 127.0, 121.8, 117.8, 51.2, 49.7, 42.5; HRMS (ESI) $m / z$ calcd for $\mathrm{C}_{27} \mathrm{H}_{23} \mathrm{~N}_{2} \mathrm{O}(\mathrm{M}+\mathrm{H})$, 391.18049, found 391.17896.

1-Benzyl-3-((4-methoxyphenyl)(phenyl)methyl)-1H-pyrrolo[2,3b]pyridin-2(3H)-one (2j): oil; $157 \mathrm{mg}(90 \%) ;{ }^{1} \mathrm{H} \mathrm{NMR}\left(\mathrm{CDCl}_{3}\right) \delta$ 8.15-8.08 (m, 2H), 7.38-7.24 (m, 6H), 7.23-7.08 (m, 10H), 7.05$6.90(\mathrm{~m}, 7 \mathrm{H}), 6.90-6.73(\mathrm{~m}, 7 \mathrm{H}), 6.67-6.59(\mathrm{~m}, 2 \mathrm{H}), 5.02-4.88(\mathrm{~m}$, $4 \mathrm{H}), 4.75(\mathrm{~d}, J=14.9 \mathrm{~Hz}, 2 \mathrm{H}), 4.39(\mathrm{t}, J=5.3 \mathrm{~Hz}, 2 \mathrm{H}), 3.80(\mathrm{~s}, 3 \mathrm{H})$, $3.74(\mathrm{~s}, 3 \mathrm{H}) ;{ }^{13} \mathrm{C} \mathrm{NMR}\left(\mathrm{CDCl}_{3}\right) \delta 175.6,175.5,158.8,158.5,157.4$, $157.3,147.3,147.2,141.4,139.5,136.3,136.3,133.0,132.6,132.5$, $130.7,129.5,129.5,128.6,128.5,128.4,127.8,127.8,127.3,127.2$, $126.9,122.1,121.9,117.8,114.0,113.8,55.4,55.2,50.7,50.4,50.0$,
49.9, 42.5; HRMS (ESI) $m / z$ calcd for $\mathrm{C}_{28} \mathrm{H}_{25} \mathrm{~N}_{2} \mathrm{O}_{2}(\mathrm{M}+\mathrm{H})$ 421.19105 , found 421.18958 .

\section{ASSOCIATED CONTENT}

\section{S Supporting Information}

Scanned NMR spectra of all new products and HPLC analysis. This material is available free of charge via the Internet at http://pubs.acs.org.

\section{AUTHOR INFORMATION}

\section{Corresponding Author}

*E-mail: marie-claude.viaud-massuard@univ-tours.fr.

Notes

The authors declare no competing financial interest.

\section{ACKNOWLEDGMENTS}

We thank the LABEX SynOrg (ANR-11-LABX-0029) and the Region Centre for financial support.

\section{REFERENCES}

(1) (a) For antitumor activity, see: Kim, K. S.; Zhang, L.; Schmidt, R.; Cai, Z.-W.; Wei, D.; Williams, D. K.; Lombardo, L. J.; Trainor, G. L.; Xie, D.; Zhang, Y.; An, Y.; Sack, J. S.; Tokarski, J. S.; Darienzo, C.; Kamath, A.; Marathe, P.; Zhang, Y.; Lippy, J.; Jeyaseelam, R., Sr.; Wautlet, B.; Henley, B.; Gullo-Brown, J.; Manne, V.; Hunt, J. T.; Fargnoli, J.; Borzilleri, R. M. J. Med. Chem. 2008, 51, 5330. (b) For antibacterial activity, see: Manchester, J. I.; Dussault, D. D.; Rose, J. A.; Ann Boriack-Sjodin, P.; Uria-Nickelsen, M.; Ioannidis, G.; Bist, S.; Fleming, P.; Hull, K. G. Bioorg. Med. Chem. Lett. 2012, 22, 5150. (c) For anti-inflammatory activity, see: Chen, G.; Liu, Z.; Zhang, Y.; Shan, X.; Jiang, L.; Zhao, Y.; He, W.; Feng, Z.; Yang, S.; Liang, G. ACS Med. Chem. Lett. 2013, 4, 69. (d) For kinase inhibition, see: Tang, J.; Hamajima, T.; Nakano, M.; Sato, H.; Dickerson, S. H.; Lackey, K. E. Bioorg. Med. Chem. Lett. 2008, 18, 4610. Hong, S.; Kim, J.; Seo, J. H.; Jung, K. H.; Hong, S.-S.; Hong, S. J. Med. Chem. 2012, 55, 5337.

(2) (a) Feringa, B. L.; Pineschi, M.; Arnold, L.; Imbos, R.; de Vries, A. H. M. Angew. Chem., Int. Ed. Engl. 1997, 36, 2620. (b) Nagaoka, Y.; Tomioka, K. Org. Lett. 1999, 1, 1467. (c) Kumagai, N.; Matsunaga, S.; Shibasaki, M. Org. Lett. 2001, 3, 4251. (d) Kantam, M. L.; Neelima, B.; Reddy, Ch. V.; Chakravarti, R. Ind. Eng. Chem. Res. 2007, 46, 8614. (e) Kumar, V.; Kaur, S.; Kumar, S. Tetrahedron Lett. 2007, 47, 7001.

(3) (a) Yang, L.; Xu, L.; Xia, C. G. Tetrahedron Lett. 2005, 46, 3279. (b) Bartoli, G.; Bosco, M.; Marcantoni, E.; Petrini, M.; Sambri, L.; Torregiani, E. J. Org. Chem. 2001, 66, 9052. (c) Loh, T. P.; Wei, L. L. Synlett 1998, 975. (d) Enders, D.; Muller, S. F.; Raabe, G.; Runsink, J. Eur. J. Org. Chem. 2000, 6, 879. (e) Varala, R.; Alam, M. M.; Adapa, S. R. Synlett 2003, 720. (f) Wabnitz, T. C.; Spencer, J. B. Tetrahedron Lett. 2002, 43, 3891. (g) Azizi, N.; Saidi, M. R. Tetrahedron 2004, 60, 383. (h) Surendra, K.; Krishnaveni, N. S.; Sridhar, R.; Rao, K. R. Tetrahedron Lett. 2006, 47, 2125. (i) Kumar, R.; Chaudhary, P.; Nimesh, S.; Chandra, R. Green Chem. 2006, 8, 356. (j) Shaikh, N. S.; Deshpande, V. H.; Bedekar, A. V. Tetrahedron 2001, 57, 9045. (k) Kantam, M. L.; Neeraja, V.; Kavita, B.; Neelima, B.; Chaudhuri, M. K.; Hussain, S. Adv. Synth. Catal. 2005, 347, 763. (1) Yang, L.; Xu, L. W.; Xia, C. G. Tetrahedron Lett. 2007, 48, 1599. (m) Xu, J. M.; Qian, C.; Liu, B. K.; Wu, Q.; Lin, X. F. Tetrahedron Lett. 2007, 63, 986. (n) Ranu, B. C.; Banerjee, S. Tetrahedron Lett. 2007, 48, 141.

(4) (a) Catalytic Asymmetric Synthesis, 2nd ed.; Wiley-VCH: New York, 2000. (b) Comprehensive Asymmetric Catalysis; Springer: New York, 1999 Vols. 1-3.

(5) (a) For general reviews on rhodium-catalyzed reactions, see: Fagnou, K.; Lautens, M. Chem. Rev. 2003, 103, 169. Yoshida, K.: Hayashi, T. In Modern Rhodium-Catalysed Organic Reactions; Evans, P. A., Ed.; Wiley-VCH: Weinheim, 2005; Chapter 3. Edwards, H. J.; Hargrave, J. D.; Penrose, S. D.; Frost, C. G. Chem. Soc. Rev. 2010, 39, 2093. (b) For 1,4-conjugate addition of aryl- and alkenylboronic acids to enones, see: Hayashi, T. Synlett 2001, 879. Hayashi, T.; Yamasaki, 
K. Chem. Rev. 2003, 103, 2829. Shintani, R.; Hayashi, T. Aldrichimica Acta 2009, 42, 31.

(6) (a) For phosphorus ligands, see: Takaya, T.; Ogasawara, T.; Hayashi, T.; Sakai, M.; Miyaura, N. J. Am. Chem. Soc. 1998, 120, 5579. Lukin, K.; Zhang, Q.; Leanna, M. R. J. Org. Chem. 2009, 74, 929. Berhal, F.; Esseiva, O.; Martin, C.; Tone, H.; Genet, J.; Ayad, T.; Ratovelomanana, V. V. Org. Lett. 2011, 13, 2806. (b) For diene ligands, see: Hayashi, T.; Ueyama, K.; Tokunaga, N.; Yoshida, K. J. Am. Chem. Soc. 2003, 125, 11508. (c) For sulfoxide ligands, see: Xue, F.; Wang, D.; Li, X.; Wan, B. J. Org. Chem. 2012, 77, 3071. Dornan, P. K.; Leung, P. L.; Dong, V. M. Tetrahedron 2011, 67, 4378.

(7) (a) Tokunaga, N.; Otomaru, Y.; Okamoto, K.; Ueyama, K.; Shintani, R.; Hayashi, T. J. Am. Chem. Soc. 2004, 126, 13584. (b) Otomaru, Y.; Okamoto, K.; Shintani, R.; Hayashi, T. J. Org. Chem. 2005, 70, 2503. (c) Shintani, R.; Kimura, T.; Hayashi, T. Chem. Commun. 2005, 3213. (d) Shintani, R.; Okamoto, K.; Hayashi, T. Org. Lett. 2005, 7, 4757. (e) Shintani, R.; Okamoto, K.; Otomaru, Y.; Ueyama, K.; Hayashi, T. J. Am. Chem. Soc. 2005, 127, 54. (f) Shintani, R.; Tsurusaki, A.; Okamoto, K.; Hayashi, T. Angew. Chem., Int. Ed. 2005, 44, 3909. (g) Nishimura, T.; Yasuhara, Y.; Hayashi, T. Org. Lett. 2006, 8, 979. (h) Chen, F.-X.; A. Kina, A.; Hayashi, T. Org. Lett. 2006, 8, 341. (i) Duan, W.-L.; Imazaki, Y.; Shintani, R.; Hayashi, T. Tetrahedron 2007, 63, 8529.

(8) Allen, J. C.; Kociok-Köhn, G.; Frost, C. G. Org. Biomol. Chem. 2012, 10, 32.

(9) Viaud, M.-C.; Jamoneau, P.; Guillaumet, G. Synth. Commun. 1997, 27, 3861.

(10) Villemin, D.; Martin, B.; Khalid, M. Synth. Commun. 1998, 28, 3195 .

(11) After semipreparative HPLC purification, each isomer of $\mathbf{1 b}$ has been isolated and analyzed over the course of a week by NMR spectroscopy in $\mathrm{CDCl}_{3}$ (the product was kept in solution during the study). For the major $E$ isomer, the evolution of the $E / Z$ ratio was as follows: $5 \mathrm{~min}$ (94/6), $3 \mathrm{~h}(90 / 10), 6 \mathrm{~h}$ (85/15), $72 \mathrm{~h}$ (81/19), 1 week $(73 / 24)$. For the minor $Z$ isomer, the evolution of the $E / Z$ ratio was as follows: $5 \mathrm{~min}$ (28/72), $3 \mathrm{~h}$ (42/58), $6 \mathrm{~h}$ (61/39), $72 \mathrm{~h}(62 / 38), 1$ week $(66 / 34)$.

(12) ChiralPak $1 \mathrm{~A}$ column, hexane/ethanol $95 / 5$ at $10{ }^{\circ} \mathrm{C}$. Retention times of the four expected stereoisomers are as follows: $21.0 \mathrm{~min}, 23.4$ min, 24.5 min (two isomers) (see the Supporting Information).

(13) ChiralPak 1A column, hexane/ethanol $95 / 5$ at $10^{\circ} \mathrm{C}$. Retention times of the two stereoisomers are as follows: $20.9 \mathrm{~min}, 24.4 \mathrm{~min}$ (see the Supporting Information).

(14) ChiralPak 1A column, hexane/ethanol $95 / 5$ at $10{ }^{\circ} \mathrm{C}$. Retention times of the two stereoisomers are as follows: $21.1 \mathrm{~min}, 24.7 \mathrm{~min}$ (see the Supporting Information).

(15) Due to the lack of reactivity with $[\mathrm{Rh}(\operatorname{cod}) \mathrm{Cl}]_{2}$ for compounds $\mathbf{2 f}$ and $\mathbf{2 h}$, it was impossible to determine an accurate ee value, even if we obtained two majors peaks (see the Supporting Information) giving, respectively, estimated ee $>80 \%$ and $\approx 88 \%$. 